\title{
Avaliação da produção de biogás a partir da co-digestão de resíduos sólidos orgânicos
}

A co-digestão anaeróbia de resíduos alimentares e grama configura-se como uma alternativa competitiva para o tratamento da fração orgânica dos resíduos sólidos urbanos, tendo em vista o contexto de insuficiente e quase nula valorização desta fração no Brasil, contrariando a legislação vigente e considerando-se, também, a típica elevada proporção de matéria orgânica no resíduos em âmbito nacional e internacional. Sob este panorama, o presente artigo buscou avaliar a produção de biogás proveniente da co-digestão de resíduos alimentares e grama esmeralda (Zoysia japonica) em 3 proporções (1:1; 2:1 e 3:1, assumindo como referência $500 \mathrm{ml}$ ) em temperatura mesofílica (38으) durante 21 dias. Avaliou-se o teor de sólidos presente na massa digerida, comparando-se com a produção de biogás e a redução de matéria orgânica biodegradável. Os resultados foram analisados estatisticamente por meio de uma análise de variância, com um nível de significância de $5 \%$. Concluída a co-digestão, o Tratamento 2 (ou seja, a proporção de 2:1 de grama em relação ao resíduo alimentar) apresentou os melhores variância, com um nivel de significância de $5 \%$. Concluida a co-digestão, o Tratamento 2 (ou seja, a proporção de 2:1 de grama em relação ao residuo alimentar) apresentou os melhores importante, pois mostra a viabilidade inerente ao uso de resíduos de grama no processo de gestão sustentável dos resíduos sólidos, norteando a proporção ideal a ser utilizada para se obte uma melhor produção de biogás. Os resultados mostram que é oportuna a realização de mais estudos, buscando otimizar ainda mais a produção de biogás, visto que a produção obtida no ensaios ainda está abaixo do potencial de geração por co-digestão de resíduos alimentares e podas de grama, conforme dados da literatura, que indicam valores da ordem de 500-600 Nm por g.SV. A produção menor de biogás observada no experimento decorre, provavelmente, do acúmulo de ácidos graxos voláteis, que inibe a ocorrência do processo de digestão por completo,

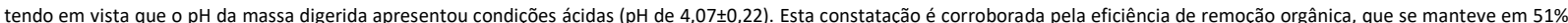
em média, para o tratamento em questão, indicando que apenas metade do potencial que poderia ser biometanizado foi, de fato, transformado em biogás.

Palavras-chave: Co-digestão anaeróbia; Resíduos alimentares; Grama esmeralda; Biogás.

\section{Evaluation of biogas production from co-digestion of organic solid}

\section{waste}

\begin{abstract}
The anaerobic co-digestion of food waste and grass is a competitive alternative for treatment of the organic fraction of urban solid waste taking into account the context of insufficient and almost null value of this fration in Brazil contrary to actual legislation and also the typical hard proportion of organic matter on wastes in national and international ambit. About this panorama the present article sought to evaluate the biogas production from the co-digestion of food waste and grass (Zoysia japonica) in 3 proportions (1:1:2:1 e 3:1 taking as reference 500 panorama the present article sought to evaluate the biogas production from the co-digestion of food waste and grass (Zoysia japonica) in 3 proportions ( $1: 1 ; 2: 1$ e $3: 1$ taking as reference 500
$\mathrm{ml}$ ) in mesophilic temperature $(38 \mathrm{o})$ ) during 21 days. The solid content present in the digested mass was evaluated, comparing to the biogas production and the reduction of biodegradable $\mathrm{ml}$ ) in mesophilic temperature $(38 \mathrm{O})$ ) during 21 days. The solid content present in the digested mass was evaluated, comparing to the biogas production and the reduction of biodegradable
organic matter. The results were analyzed statistically through a variance analysis considering $5 \%$ of significance. Co-digestion done, treatment 2 (in other words, the proportion of 2:1 of grass in relation to food waste) presented the best results in terms of biogas generation. It results in maximum biogas production, which was $1440 \mathrm{Nml}$ of accumulated biogas or $25 \mathrm{Nml}$ pe gram of volatile solid (VS). These results mean an important verification, because it shows the inherent viability of the use the of grass clippings in the process of sustainable management of solids wastes, guiding the ideal proportion to be used in order to obtain a better biogas production. The results show that further researches are needed for optimize the biogas production since the production obtained during the test is still below the potential of biogas generation through co-digestion with food waste and grass clippings which, according to the literature, indicates values of 500-600 Nml per a.VS. The lower biogas production observed during the experiments is probably due to the accumulation of volatile acids, which inhibits the occurrence of the whole digestion process bearing in mind that the $\mathrm{pH}$ of digestate mass presents acid conditions $(4.07 \pm 0.22)$. This finding is corroborated by the efficiency of organic removal, which remained on average $51 \%$ on average for the treatment in question, indicating that only half of potential that could be biomethanized was, in fact, turned into in biogas.
\end{abstract}

Keywords: Anaerobic co-digestion; Food waste; Emerald grass; Biogas.

Topic: Engenharia Sanitária

Reviewed anonymously in the process of blind peer.
Received: 05/03/2021

Approved: 24/03/2021
Fernanda de Marco de Souza (1D)

Universidade de São Paulo, Brasil http://lattes.cnpq.br/5342355090074447

http://orcid.org/0000-0002-9263-8735

fernanda.marco.souza@usp.br

Joyce Meneses da Silva Jaeger (iD

Universidade de São Paulo, Brasil

http://lattes.cnpq.br/5300304875725665

http://orcid.org/0000-0003-4754-7751

joycejaeger11@gmail.com

Ednilson Viana (iD)

Universidade de São Paulo, Brasil

http://lattes.cnpq.br/7928208403989434

http://orcid.org/0000-0002-8483-4082

ednilson.viana@gmail.com

\author{
Giovano Candiani (iD \\ Universidade Federal de São Paulo, Brasil \\ http://lattes.cnpq.br/9950995765229751 \\ http://orcid.org/0000-0001-9896-4390 \\ giovanocandiani@gmail.com \\ André Felipe Simões \\ Universidade de São Paulo, Brasil \\ http://lattes.cnpq.br/1503283535579534 \\ http://orcid.org/0000-0002-4108-9596 \\ afsimoes@usp.br \\ Homero Fonseca Filho (iD \\ Universidade de São Paulo, Brasil \\ http://lattes.cnpq.br/8291933997449674 \\ http://orcid.org/0000-0003-4737-9938 \\ homeroff@usp.br
}

\section{Referencing this:}

SOUZA, F. M.; JAEGER, J. M. S.; VIANA, E.; CANDIANI, G.; SIMOES, A. F.; FONSECA FILHO, H.. Avaliação da produção de biogás a partir da codigestão de resíduos sólidos orgânicos. Revista Ibero Americana de Ciências Ambientais, v.12, n.3, p.299-311, 2021. DOI: http://doi.org/10.6008/CBPC2179-6858.2021.003.0025 


\section{INTRODUÇÃO}

A geração anual de resíduos sólidos urbanos (RSU) no Brasil é da ordem de 79 milhões de toneladas (ABRELPE, 2019). Do total coletado (que corresponde a 91,2\% da geração), quase a totalidade é encaminhada para disposição final em solo - aterros sanitários, aterros controlados e lixões - de modo que apenas pouco mais de $5 \%$ possui algum tipo de valorização através de unidades de triagem e compostagem (SNIS, 2019).

Tal panorama revela que o Brasil está na contramão do que a Política Nacional de Resíduos Sólidos PNRS (expressa na Lei no 12.305/2010) preconiza e estabelece como ordem de prioridade na gestão e gerenciamento de resíduos sólidos, conforme o art. 9ํ, o qual indica: não geração, redução, reutilização, reciclagem, tratamento dos resíduos sólidos e disposição final ambientalmente adequada dos rejeitos. A PNRS também estabelece que apenas os rejeitos devem ser destinados para disposição final ambiental adequada (aterros sanitários), de modo que devem ser esgotadas todas as possibilidades de tratamento e recuperação por processos tecnológicos disponíveis e economicamente viáveis antes que os resíduos sejam dispostos em solo (BRASIL, 2010). Somado a isso, cerca de $50 \%$ da massa dos RSU é constituída por fração orgânica - resíduos alimentares, resíduos de jardinagem, madeira etc. (ABRELPE, 2019; SNIS, 2019), passível de ser submetida a tecnologias de tratamento e valorização.

Além disso, a elevada geração e a disposição ambientalmente inadequada dos resíduos configuramse como importantes aspectos ambientais que acarretam múltiplas externalidades ambientais negativas que precisam ser consideradas no seio de uma gestão endereçada à sustentabilidade, com fins de mitigar a excessiva depleção em curso dos recursos naturais do planeta, assim como reduzir os riscos à saúde humana e às demais espécies vivas e atenuar os passivos ambientais (KHALID et al., 2011).

É neste contexto de insuficiente e quase nula valorização dos RSU no Brasil, contrariedade à legislação vigente e elevada proporção de matéria orgânica, alinhado à uma perspectiva de gestão sustentável de resíduos sólidos, que se insere o processo de digestão anaeróbia como uma tecnologia competitiva no gerenciamento dos resíduos. É sob tal égide também que emerge, em boa medida, a justificativa para realização do presente trabalho.

A digestão anaeróbia (DA) é um processo de degradação e estabilização da matéria orgânica na ausência de oxigênio, no qual diversas comunidades microbianas interagem na conversão da fração biodegradável em biogás (composto, principalmente, por metano) e matéria orgânica digerida, que pode ser utilizada como biofertilizante, com alto teor de nutrientes (CHEN et al., 2008; KWIETNIEWSKA et al., 2014; WU, 2019).

A DA se aplica a uma elevada diversidade de materiais, incluindo os resíduos verdes (biomassa lignocelulósica, tais como folhas de árvores e podas de grama, em especial) e alimentares, presentes nos RSU (KHALID et al., 2011), de modo que pode ser executada com somente um substrato ou mais (co-digestão). A co-digestão é utilizada quando se objetiva equilibrar nutrientes (relação $\mathrm{C} / \mathrm{N}$, macro e micronutrientes) e aumentar a produtividade de metano (ZHU et al., 2014). Nisto, tem-se que os resíduos de alimentos e de verdes - como a grama - possuem alto potencial de complementariedade (BROWN et al., 2013). 
Em conjunto com os resíduos domiciliares orgânicos, a biomassa lignocelulósica tem sido amplamente utilizada na produção de biogás nos últimos anos (BEDOIĆ, 2019), tornando-se uma promissora matéria prima associada aos restos alimentares.

Outrossim, do ponto de vista energético, o biogás gerado possui alto potencial para ser utilizado como fonte de energia (ICLEI, 2010), e se apresenta como uma energia limpa e renovável (CURTO et al., 2019), que pode produzir eletricidade, calor e até mesmo servir como combustível para veículos, com chances de substituir o gás natural (SCARLAT, 2018), contribuindo para a redução da emissão antrópica de gases do efeito estufa (FEROLDI et al., 2018; COIMBRA-ARAÚJO et al., 2014; SHANE et al., 2018).

Diante de um quadro de necessária e urgente transição de uma matriz energética centrada nos combustíveis fósseis para uma outra matriz tipicamente bem menos carbono-intensiva e, assim, centrada em fontes de energia renováveis e, também, em vista das necessárias preocupações com a segurança energética, o biogás se apresenta como uma fonte renovável e limpa (LOPES et al., 2018; MAUGER, 2019).

Do ponto de vista energético, o biogás, que é rico em metano (50-75\% de sua composição), é fonte de energia térmica, elétrica, cinética e luminosa (ICLEI, 2010). Estima-se que, pelo menos, $25 \%$ de toda a bioenergia pode ser derivada deste gás produzido durante a digestão anaeróbia da fração orgânica de resíduos e efluentes (HOLM-NIELSEN et al., 2009).

De acordo com Mao et al. (2015), a produção do biogás não somente reduz os custos para o tratamento dos resíduos, como também possui uma matéria-prima de baixo custo, uma vez que é gerado a partir de substratos antes considerados como "lixo".

Verifica-se, portanto, que uma das principais vantagens do biogás está na eliminação dos resíduos orgânicos presentes nas categorias industriais, domésticos e misturas de orgânicos, que estão presentes no contexto do espaço urbano. É com base neste diferencial positivo correlato ao biogás e nos diversos outros benefícios (que a digestão anaeróbia e seus produtos fornecem para as populações humanas e não humanas), e na importância de se investir em energias renováveis e realizar uma gestão de resíduos endereçada à sustentabilidade que estudos na área estão sendo, cada vez mais, desenvolvidos.

Deste modo, dado o contexto de necessária gestão e gerenciamento sustentável dos resíduos sólidos, aliado à uma elevada disponibilidade de resíduos verdes, (como a grama) e uma fração de resíduos orgânicos correspondente a mais da metade do que é gerado de RSU, a presente pesquisa buscou investigar quantitativamente a produção de biogás a partir da co-digestão de resíduos alimentares e grama.

\section{MATERIAS E MÉTODOS}

\section{Substratos e inóculo}

O ensaio para determinar a produção de biogás, a partir da co-digestão de resíduos sólidos orgânicos foi realizado no laboratório experimental do Centro de Valorização de Resíduos Sólidos na Escola de Artes, Ciências e Humanidades da Universidade de São Paulo (EACH-USP - também conhecida como 'USP LESTE'). Os resíduos alimentares (com predomínio de frutas, verduras e legumes, os quais foram triturados 
em um liquidificador até atingir consistência pastosa, a fim de aumentar a área de contato e acelerar as reações químicas da digestão anaeróbia.

Para a avaliação da co-digestão, além do uso dos resíduos alimentares foram utilizados, também, como substratos os resíduos de poda de grama esmeralda (Zoysia japonica), produzidos no campus universitário (EACH-USP) a partir de atividades associadas à manutenção das suas áreas verdes. A grama foi picada manualmente até atingir comprimentos de 2-3 cm, também com o intuito de gerar maior superfície de contato.

A grama usada foi em função de que materiais lignocelulósicos podem ser uma fonte de inóculo (ALVES, 2016), além de ser uma oportunidade de testar seu efeito no processo de co-digestão, permitindo avaliar se é possível fazer o gerenciamento desse resíduo por esta via, uma vez que é um resíduo produzido com grande volume no campus universitário.

Além destes substratos, o lodo coletado de um reator UASB (Upflow Anaerobic Sludge Blanket) de uma Estação de Tratamento de Esgoto (ETE) foi utilizado como inóculo.

\section{Aparatos e reagentes experimentais}

Para a medição da produção de biogás, realizou-se o ensaio com base nas normas alemãs VDI 4630 e DIN 38414 (S8), optando-se pela leitura em tubos eudiômetros (digestores) de 500 ml, com graduação de $1 \mathrm{ml}$, da marca FGG e calibração da Rede Brasileira de Calibração (RBC), acoplados a frascos reatores de $1 \mathrm{~L}$, em vidro (Figura 1), vedados com graxa a base de Silicone Molykote HVG.

Neste sistema, a quantidade de biogás produzida é obtida através da visualização do deslocamento do líquido selante contido no eudiômetro graduado. A leitura do biogás deve ser feita igualando o menisco do líquido selante do tubo eudiômetro com o menisco do frasco reservatório, para que apenas a pressão atmosférica esteja incidindo na coluna de biogás. Caso a medição seja realizada a partir do nível 0 (zero) do eudiômetro, deve-se ajustar uma equação de reta para a produção de biogás e aplicar nos valores lidos a partir do líquido deslocado. Com vistas a facilitar o manuseio das vidrarias, optou-se por ajustar a equação, através do método de regressão linear $\left(y=1,2292 x-0,4171\right.$, com $R^{2}$ de 0,989$)$.

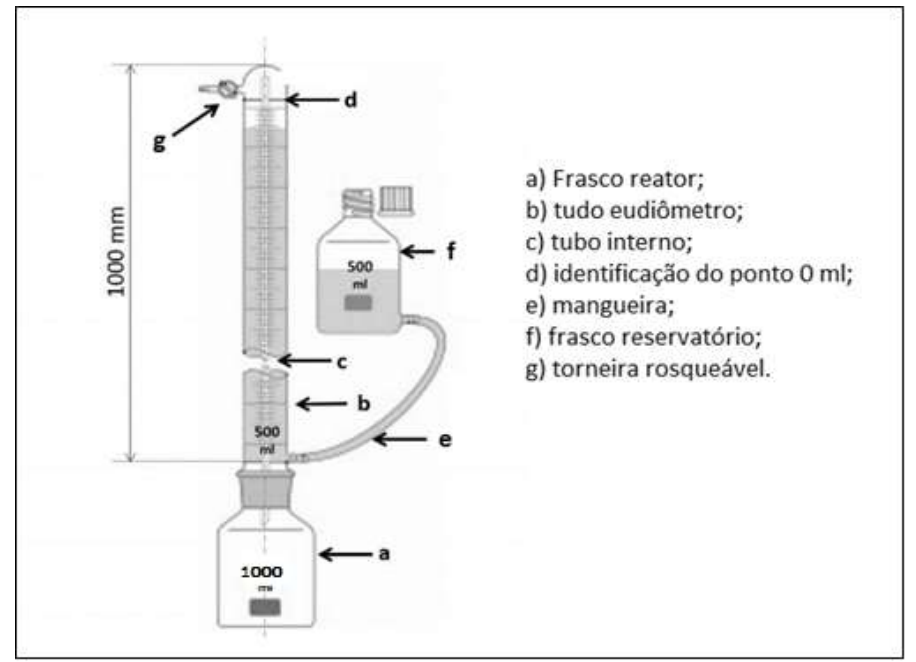

Figura 1: Esquema do conjunto eudiômetro utilizado no experimento. Fonte: Adaptado de Lima (2016). 
No que concerne à composição do líquido selante, este consiste em uma solução ácida que evita a solubilização do dióxido de carbono presente no biogás. Para tal, a solução foi preparada a partir de $200 \mathrm{~g}$ de sulfato de sódio decahidratado $\left(\mathrm{Na}_{2} \mathrm{SO}_{4} * 10 \mathrm{H}_{2} \mathrm{O}\right)$ e $30 \mathrm{ml}$ de ácido sulfúrico $\left(\mathrm{H}_{2} \mathrm{SO}_{4}\right.$, concentração $98 \%$ e densidade $1,84 \mathrm{~g} / \mathrm{cm}^{3}$ ), para cada $1000 \mathrm{ml}$ de água destilada.

A mensuração da produção de biogás no trabalho, seguindo as normas alemãs - VDI 4630 e DIN 38 414 (S8), foi realizada utilizando-se o conjunto eudiômetro considerando um período de digestão de 21 dias (LIMA, 2016). Durante este período, diariamente a produção de biogás foi aferida no mesmo horário, para completar ciclos de $24 \mathrm{~h}$ cada. O biogás medido foi corrigido nas Condições Normais de Temperatura e Pressão (CNTP), conforme aponta a norma alemã, VDI 4630, utilizando-se a Equação 1.

$$
V_{0}=\mathrm{V} \cdot \frac{\left(P_{L}-P_{W}\right) \cdot T_{0}}{P_{0} \cdot \mathrm{T}}
$$

$$
\begin{array}{r}
\text { Onde, } \\
V_{0}=\text { volume de biogás em condições normais }(\mathrm{mL}) ; \\
V=\text { volume registrado no eudiômetro }(\mathrm{mL}) ; \\
P_{L}=\text { pressão atmosférica no momento do registro }(\mathrm{mbar}) ; \\
\mathrm{PW}_{\mathrm{W}}=\text { pressão do vapor d’água }(\mathrm{mbar}) ; \\
\mathrm{T}_{0}=\text { temperatura normal }(273 \mathrm{~K}) ; \\
\mathrm{P}_{0}=\text { pressão normal }(1.013 \mathrm{mbar}) ; \\
\mathrm{T}=\text { temperatura }(\mathrm{K}) .
\end{array}
$$

Além disso, a pressão de vapor d'água (que corresponde à pressão exercida pelas moléculas de água gasosa) pode levar à superestimação dos valores do volume de biogás em condições normais, para evitar tal ocorrência, utilizou-se a Equação 2 (STRÖMBERG et al., 2014).

$$
P_{W}=10^{8,1962-\left(\frac{1730,63}{T-39,724}\right)}
$$

Onde,

$\mathrm{Pw}_{\mathrm{w}}$ pressão do vapor d'água (mbar); $\mathrm{T}=$ temperatura $(\mathrm{K})$.

A temperatura e pressão atmosférica foram tomadas a partir de uma estação meteorológica instalada no interior do laboratório. Para tal medição, utilizou-se uma Estação Gaia T-EST-0056 com Calibração Termo Higrômetro Rastreável.

\section{Delineamento experimental}

A Tabela 1 mostra os três tratamentos utilizados com diferentes proporções em unidades de volume. Cada tratamento foi realizado em triplicata ( $n=3$ ), conforme Faisal et al. (2020) e Ketsub et al. (2021). A razão entre inóculo e substrato, de acordo com a norma alemã VDI 4630, é estabelecida com base em sólidos voláteis (SV), de maneira que a razão entre a quantidade de SV do substrato por inóculo deve ser de 0,5.

Tabela 1: Proporção das triplicatas na avaliação da co-digestão.

\begin{tabular}{lllll}
\hline & Tratamento 1 & Tratamento 2 & Tratamento 3 & Tratamento 4 \\
\cline { 2 - 5 } Resíduos alimentares & $250 \mathrm{ml}$ & $333 \mathrm{ml}$ & $375 \mathrm{ml}$ & - \\
\hline Grama & $250 \mathrm{ml}$ & $166 \mathrm{ml}$ & $125 \mathrm{ml}$ & - \\
\hline Inóculo & $250 \mathrm{ml}$ & $250 \mathrm{ml}$ & $250 \mathrm{ml}$ & $250 \mathrm{ml}$ \\
\hline
\end{tabular}

Os digestores (Figura 1) em escala de bancada, contendo os substratos, foram mantidos em banho- 
maria, com a utilização de aquários de vidro e um aquecedor de água, que manteve a temperatura constante em $38 \pm 1$ 으 (Figura 2), classificando o processo na faixa mesofílica. $\mathrm{O} \mathrm{pH}$ da mistura dos substratos foi corrigido para próximo da neutralidade (por meio da adição de hidróxido de magnésio), com o intuito de facilitar o processo de digestão anaeróbia.

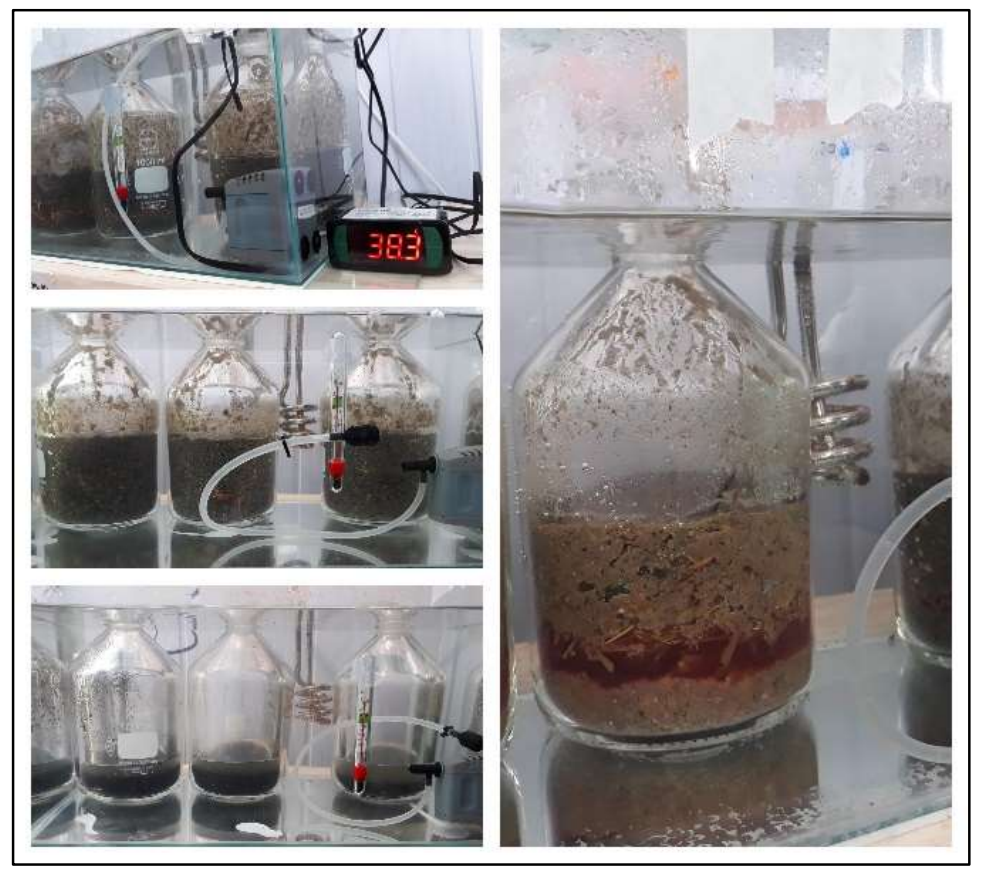

Figura 2: Disposição dos digestores em aquários de vidro sob temperatura constante.

\section{Teste de sólidos}

Aliado à verificação da produção de biogás, também se realizou um teste de sólidos sob os substratos (grama e resíduos alimentares) e digerido com o intuito de correlacionar a quantidade de sólidos voláteis (passível de ser volatizado) e a produção de biogás. Para aferição da umidade, sólidos totais e voláteis da amostra, utilizou-se o método padrão da American Water Works Association (APHA, 2005). Tal método estabelece que os sólidos totais são obtidos após secagem em estufa a 105드 até atingir pesos constantes e posteriormente são calcinadas em mufla a uma temperatura de 550 으 por $2 \mathrm{~h}$ para determinação da quantidade de sólidos voláteis.

\section{Tratamento dos dados}

Os resultados obtidos com o ensaio da co-digestão, foram analisados estatisticamente por meio de um teste de Análise de Variância (ANOVA), utilizando-se o programa computacional MINITAB (2019), verificando-se a existência de diferenças entre os diferentes tratamentos empregados, a um nível de significância de $5 \%$.

\section{RESULTADOS E DISCUSSÃO}

\section{Análise do biogás proveniente da co-digestão}

Finalizados os 21 dias do processo de digestão anaeróbia, a produção acumulada da geração diária 
de biogás atingiu cerca de $1.800 \mathrm{Nml}$ (Figura 3), no qual ' $\mathrm{N}$ ' configura-se como Normal, unidade do Sistema Internacional que se refere às condições normais de temperatura e pressão, CNTP (LIMA, 2016).

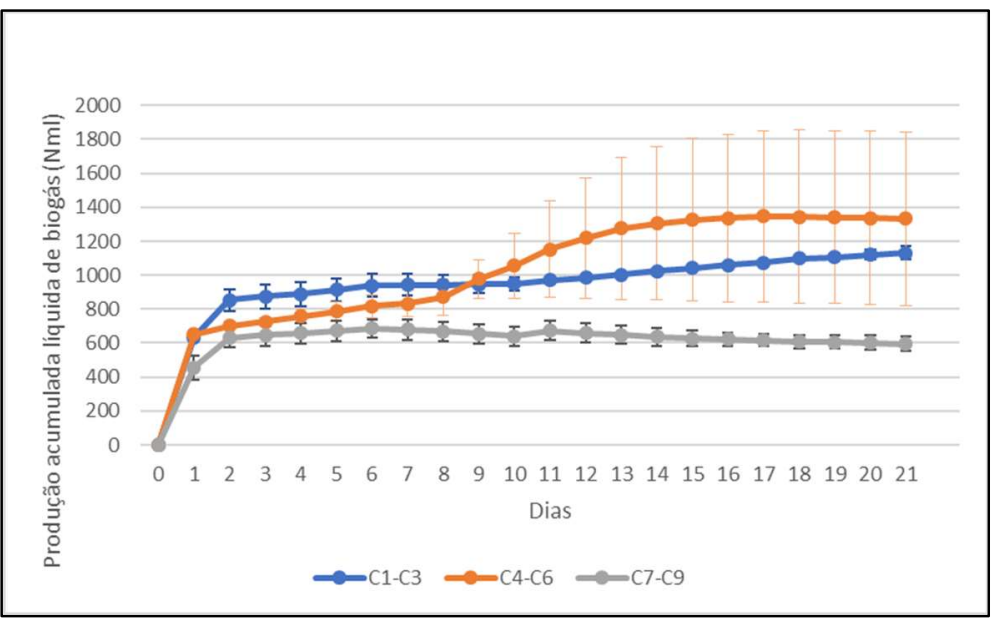

Figura 3: Produção acumulada líquida de biogás, onde C1-C3 corresponde ao tratamento 1, C4-C6 ao tratamento 2 e C7-C9 ao 3.

A partir dos resultados, verificou-se que entre os três tratamentos, aquele que apresentou os maiores valores de geração de biogás foi o 2 (C4-C6 na Figura 3), com $333 \mathrm{ml}$ de resíduos e $166 \mathrm{ml}$ de grama (proporção de 2: 1, assumindo $500 \mathrm{ml}$ como referência).

Para verificar se a diferença constatada entre os tratamentos é significativa, torna-se necessário observar os resultados estatísticos. A partir da verificação do valor-p de 8\% para o teste $\mathrm{F}$ (conforme a Tabela 2), rejeita-se a hipótese nula, indicando que existe diferença entre as médias verificadas. Ressalta-se que o ideal seria um valor-p de até $5 \%$, mas até $10 \%$ se pode rejeitar a hipótese em questão.

Tabela 2: Análise de Variância do biogás versus tratamento.

\begin{tabular}{llllll}
\hline Fonte & GL & SQ & QM & Valor $\mathbf{F}$ & Valor-p \\
\hline Tratamento & 2 & 383171 & 191586 & 3,75 & 0,088 \\
Erro & 6 & 306919 & 51153 & & \\
Total & 8 & 690090 & & & \\
\hline
\end{tabular}

Fonte: Elaborado a partir de MINITAB (2019).

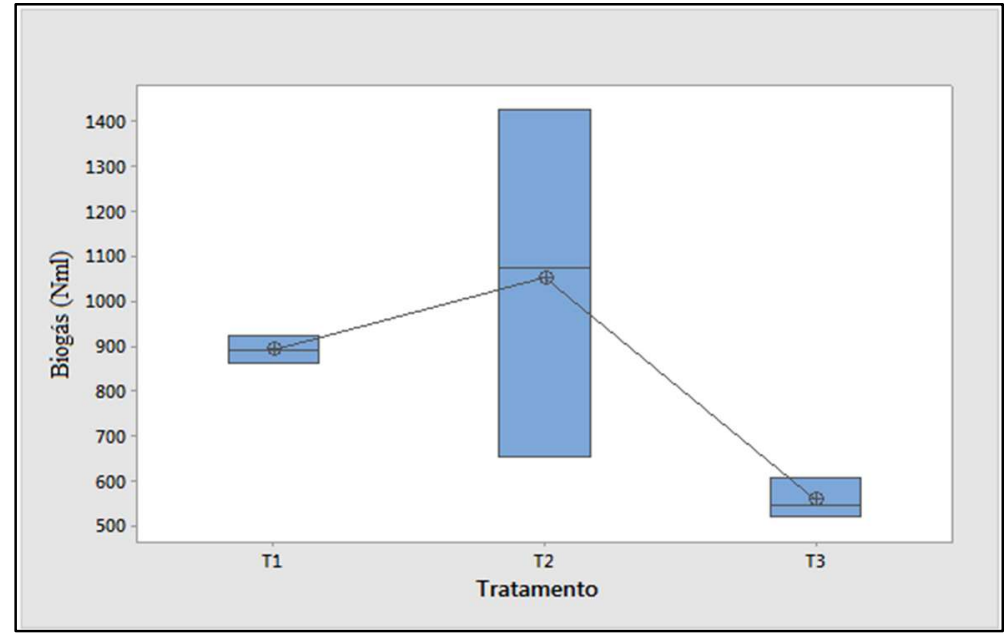

Figura 4: Boxplot da produção de biogás por tratamento utilizado, onde: T1: Tratamento 1; T2: Tratamento 2 e T3: Tratamento 3. Fonte: Elaborado a partir de MINITAB (2019).

Com base no Boxplot (Figura 4) gerado a partir da produção de biogás dos três tratamentos, tem-se 
que o Tratamento 2 é aquele que apresenta maior variabilidade, mas, ainda assim, possui uma média de produção maior do que os outros dois tratamentos testados. Além disso, obteve-se 0,5552 de determinação, indicando que o tratamento empregado e a produção de biogás estão 55,52\% associados.

Sobre a quantidade de sólidos totais (ST) e voláteis (SV) dos substratos obtida no teste de sólidos, estes estão amparados pela literatura, indicando que os resíduos alimentares possuem de $15-20 \%$ de ST e de 85-95\% de sólidos voláteis relativos aos sólidos totais (SCHIEVANO et al., 2008). Por sua vez, os valores verificados para a grama estão próximos aos obtidos por Andriamanohiarisoamanana et al. (2017) e Brown et al. (2012), que apresentam 23,075\% de ST e 91,7\% de SV, respectivamente. De acordo com a Tabela 3, é possível visualizar os resultados dos substratos empregues no ensaio de digestão.

Tabela 3: Características dos substratos utilizados no ensaio $(n=3)$.

\begin{tabular}{lll}
\hline Parâmetros & Resíduo alimentar & Grama \\
\hline ST (\%) & $15,18 \pm 1,49$ & $34,62 \pm 3,82$ \\
SV (\%ST) & $92,81 \pm 0,39$ & $90,56 \pm 0,94$ \\
\hline
\end{tabular}

Ao analisar do ponto de vista do teor de SV, pode-se verificar a relação destes com os tratamentos aplicados para a co-digestão. Progressivamente, seguindo o aumento na quantidade de resíduos alimentares na mistura, tem-se também o aumento dos SV em termos absolutos, uma vez que o resíduo verde utilizado ocupa uma grande quantidade em volume, com baixa densidade. Assim, a quantidade de ST e voláteis presentes na mistura corresponde, quase que em totalidade, à quantidade de resíduos alimentares (Tabela $4)$.

Tabela 4: Quantidade de sólidos voláteis nos tratamentos utilizados no ensaio.

\begin{tabular}{llll}
\hline & Tratamento 1 & Tratamento 2 & Tratamento 3 \\
\hline Resíduos alimentares $\mathbf{( g )}$ & 38,880 & 51,788 & 58,320 \\
Grama (g) & 5,486 & 3,642 & 2,484 \\
Valor absoluto (g) & 44,366 & 55,431 & 60,804 \\
Valor relativo à quantidade total (\%) & 0,156 & 0,150 & 0,147 \\
\hline
\end{tabular}

Quando se verifica o valor absoluto de SV em função da quantidade de biogás produzida, obtém-se a relação média do biogás por grama de sólido volátil (g.SV) e, deste modo, tem-se o quanto cada grama de matéria orgânica produziu em média de biogás durante o período analisado (Figura 5).

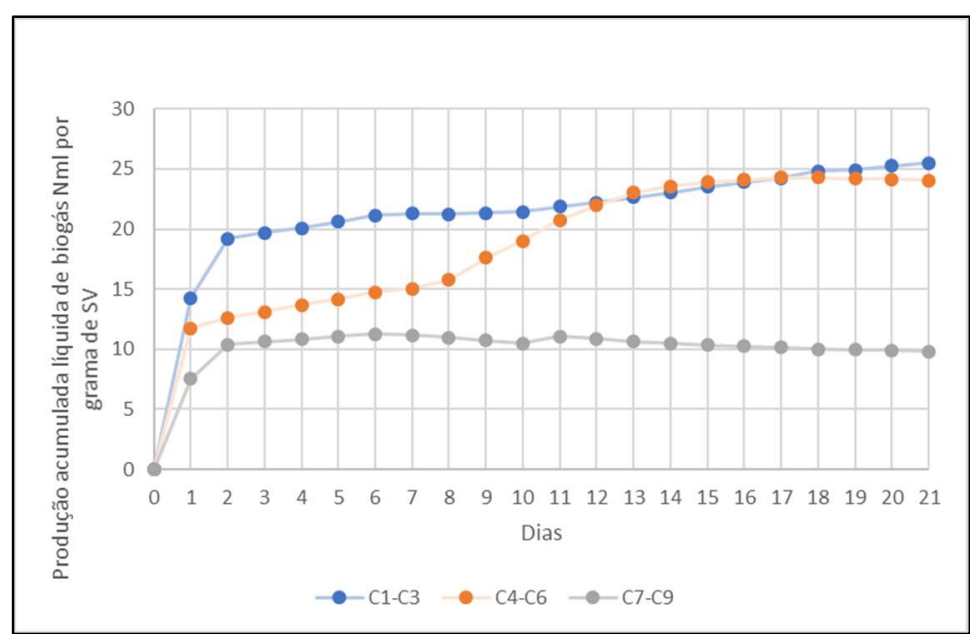

Figura 5: Produção acumulada líquida de biogás por grama de sólidos voláteis, onde C1-C3 corresponde ao tratamento 1, C4-C6 ao tratamento 2 e C7-C9 ao 3. 
É possível visualizar que a maior quantidade de biogás produzida em média é de, aproximadamente, $25 \mathrm{Nml}$ por g.SV adicionados (Figura 5). Entretanto, este valor é baixo quando comparado ao que se têm para os potencias de produção de biogás e resíduos alimentares e grama, que estão numa faixa de 500-600 Nml/g.SV (MATTIOLI et al., 2017; ARIUNBAATAR et al., 2014). Tal valor pode ser decorrente de uma possível inibição da degradação, causada por uma elevada presença de ácidos graxos voláteis na massa de resíduos

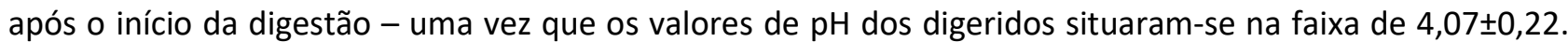
Esse valor é inferior ao que se espera para a massa digerida, que deve estar em torno de 7,5 conforme indica uma extensa revisão realizada por Beggio et al. (2019).

Esta inibição pode estar relacionada com a maior digestibilidade dos resíduos alimentares (que são facilmente degradados), se comparados aos resíduos lignocelulósicos. Este comportamento leva a uma elevada produção de ácidos graxos voláteis (AGVs), que inibe a metanogênese (etapa da digestão anaeróbia, onde se tem a produção acelerada de metano), conforme indica Chen et al. (2014), que delinearam um ensaio semelhante ao executado. Após o início da degradação, o produto inicial solubilizado da fração orgânica é imediatamente usado pelas bactérias anaeróbias e convertidos em AGVs (KAWAl et al., 2014). Somado a uma maior digestibilidade dos resíduos alimentares, a alta carga de sólidos (adotada para este ensaio) pode favorecer uma reprodução rápida das bactérias no estágio inicial da digestão, aumentando também o quanto se produz de ácidos (ZHANG et al., 2014). A constatação numérica (25 Nml/g.SV) também vai ao encontro dos dados inerentes às figuras 3 e 5, nas quais é possível verificar que a taxa de produção de biogás se estabiliza após o primeiro pico do sistema, entre os dias 1 e 2 .

A não completude da digestão é corroborada pela redução no teor de sólidos voláteis, que se manteve em 51,91\%; 51,34\% e até mesmo 38,08\% para os tratamentos 1, 2 e 3 (Tabela 5), respectivamente; de maneira que a degradação da matéria orgânica poderia ter sido mais efetiva, tendo em vista que apenas metade daquilo que poderia ser biometanizado através da ação das bactérias foi, de fato, volatizado, conforme se observa na Tabela 5. Desta maneira, métodos e substâncias para controle do pH durante a digestão devem ser empregados com o objetivo de tamponar a mistura e evitar a acidificação.

Tabela 5: Reduções nas quantidades de sólidos totais e voláteis por grama de amostra, no qual STi e SVi correspondem aos sólidos iniciais e STf e SVf, aos finais.

\begin{tabular}{|c|c|c|c|c|c|c|c|c|}
\hline \multirow{2}{*}{$\begin{array}{l}\text { Digerido } \\
\text { Tratamento } 1\end{array}$} & \multicolumn{4}{|c|}{ ST (g/g de amostra) } & \multicolumn{4}{|c|}{ SV (g/g de amostra) } \\
\hline & STi & STf & Redução (g) & Redução (\%) & SVi & SVf & Redução (g) & Redução (\%) \\
\hline $\mathrm{C} 1$ & \multirow[t]{3}{*}{0,166} & 0,093 & 0,0727 & 43,90 & \multirow[t]{3}{*}{0,1538} & 0,0759 & 0,0779 & 50,65 \\
\hline $\mathrm{C} 2$ & & 0,088 & 0,0777 & 46,92 & & 0,0735 & 0,0803 & 52,21 \\
\hline C3 & & 0,088 & 0,0779 & 47,04 & & 0,0725 & 0,0813 & 52,86 \\
\hline Média & & 0,0895 & 0,0761 & $45,95 \%$ & & 0,07397 & 0,0798 & $51,91 \%$ \\
\hline Tratamento 2 & STi & STf & Redução (g) & Redução (\%) & SVi & SVf & Redução (g) & Redução (\%) \\
\hline $\mathrm{C} 4$ & \multirow[t]{3}{*}{0,16} & 0,08 & 0,0803 & 50,16 & \multirow[t]{3}{*}{0,149} & 0,0692 & 0,0798 & 53,56 \\
\hline $\mathrm{C} 5$ & & 0,087 & 0,0732 & 45,72 & & 0,0796 & 0,0694 & 46,58 \\
\hline C6 & & 0,077 & 0,0829 & 51,78 & & 0,0687 & 0,0803 & 53,89 \\
\hline Média & & 0,0813 & 0,0788 & $49,22 \%$ & & 0,0725 & 0,0765 & $51,34 \%$ \\
\hline Tratamento 3 & STi & STf & Redução (g) & Redução (\%) & SVi & SVf & Redução (g) & Redução (\%) \\
\hline $\mathrm{C7}$ & \multirow[t]{3}{*}{0,158} & 0,106 & 0,0522 & 32,98 & \multirow[t]{3}{*}{0,1474} & 0,09 & 0,0574 & 38,94 \\
\hline $\mathrm{C} 8$ & & 0,111 & 0,0478 & 30,2 & & 0,094 & 0,0534 & 36,23 \\
\hline C9 & & 0,108 & 0,0504 & 31,84 & & 0,0898 & 0,0576 & 39,08 \\
\hline Média & & 0,1082 & 0,0501 & $31,67 \%$ & & 0,09127 & 0,0561 & $38,08 \%$ \\
\hline
\end{tabular}


Os resultados obtidos com digeridos provenientes dos nove reatores em escala de bancada também foram submetidos à análise de variância (ANOVA). O valor-p verificado, abaixo de 5\%, também indica que existe diferença entre as médias, contribuindo para que depreenda, para efeito de conclusão, que os tratamentos empregados foram de fato substanciais para gerar resultados significativamente diferentes (Tabela 6). Frisa-se, neste contexto, que o coeficiente de determinação foi de 0,8754, o que indica que o teor de SV e o tratamento empregado estão $87,54 \%$ associados.

Tabela 6: ANOVA g de SV/g de amostra versus tratamento.

\begin{tabular}{llllll}
\hline Fonte & GL & SQ & QM & Valor $\mathbf{F}$ & Valor-p \\
\hline Tratamento & 2 & 0,000654 & 0,000327 & 21,07 & 0,002 \\
Erro & 6 & 0,000093 & 0,000016 & & \\
Total & 8 & 0,000747 & & & \\
\hline
\end{tabular}

Fonte: Elaborado a partir de MINITAB (2019).

\section{Potenciais socioambientais do biogás}

Do ponto de vista energético, social e ambiental, a valorização dos resíduos orgânicos através de digestores anaeróbios (em grandes ou pequenas usinas), encerra fomento à democratização e à universalização do acesso à energia, viabilizando fornecimento até as comunidades mais afastadas dos centros urbanos (como, por exemplo, comunidades isoladas na Amazônia) e que ainda não possuem acesso à rede elétrica, principalmente.

Desta forma, sob um ângulo mais amplo, considerando a Agenda 2030 para o desenvolvimento sustentável, o biogás pode ser um caminho para que os espaços urbanos se aproximem do Objetivo do Desenvolvimento Sustentável (ODS) 7, no qual se almeja assegurar o acesso confiável, sustentável, moderno e a preço acessível à energia para todos (ONU, 2015). Indo além, o biogás gerado a partir da valorização dos resíduos, possui capacidade para substituir o gás natural (para uso como combustível veicular, por exemplo), aproveitando a infraestrutura de transporte já existente (CURTO et al., 2019) - diminuindo, assim, os custos econômicos correlatos a seu emprego.

Um ponto estratégico para a utilização do biogás, é que ele pode ser gerado através das pequenas usinas, como as domiciliares, e isto pode descentralizar a geração de energia. Considerando um país com dimensões continentais, como o Brasil, a descentralização pode ser um forte aliada para solucionar problemas socioambientais, uma vez que o tratamento destes na fonte, em geral, fornece retorno imediato às comunidades afastadas, assim como pode ser aplicado ao contexto urbano; configurando-se, portanto, o biogás num agente que promove a acessibilidade energética. Também vale considerar que a descentralização energética aumenta a segurança energética, reduzindo assim as vulnerabilidades (LUCON et al., 2009).

Considerando o atual modelo de gestão dos resíduos sólidos brasileiro que preza pela destinação final em solo, em detrimento da não geração, reutilização e reciclagem (SNIS, 2019), a digestão anaeróbia se insere como uma forma para alterar este quadro, valorizando até $50 \%$ do que se tem para os resíduos domiciliares; e, assim, contribuindo para o cumprimento dos objetivos da Política Nacional de Resíduos Sólidos, art. 7으, inciso II e o inciso IV, que prevê a "adoção, desenvolvimento e aprimoramento de tecnologias limpas como forma de minimizar impactos ambientais" (BRASIL, 2010). 
Tomando tal citação como base, do ponto de vista ambiental, dentro de um contexto onde se tem uma crescente produção de resíduos sólidos urbanos e no qual as emissões de gás metano representam pelo menos 21 vezes mais potencial, que o dióxido de carbono na intensificação do efeito estufa terrestre (BAIRD et al., 2011), e que os aterros sanitários são uma das maiores fontes antropogênicas deste gás (CHARLES et et al., 2009), é de suma importância que melhores práticas de gerenciamento dos resíduos sejam realizadas.

Assim, a digestão anaeróbia e o correlato potencial de co-digestão apresentados surgem como uma alternativa competitiva para o tratamento dos resíduos orgânicos, além de se configurar numa fonte energética renovável, confiável (sob o prisma da previsibilidade produtiva) e limpa (quando comparada aos combustíveis fósseis, em especial).

Considerando-se os resultados obtidos da produção de biogás neste trabalho, ressalta-se que estes são provenientes de ensaio em escala de bancada, devendo-se, portanto, ser analisado com ressalvas em um contexto de uma escala real, em que as condições de controle são mais difíceis e complexas. Assim, se faz necessário adequar as potencialidades em termos da produção de biogás de sistemas de co-digestão anaeróbia, frente aos investimentos necessários para sua devida implementação em uma escala real, sobretudo no que concerne ao retorno financeiro planejado.

\section{CONCLUSÕES}

Os resultados obtidos indicam que a proporção de 2:1, ou seja, $166 \mathrm{ml}$ de resíduos de grama esmeralda em associação com os $333 \mathrm{ml}$ resíduos alimentares apresenta maior potencial relativo de geração de biogás (tratamento 2), atingindo $1.400 \mathrm{Nml}$ gerados em média e até $1.800 \mathrm{Nml}$ em um dos conjuntos reatores presentes na triplicata. Esse dado é corroborado por análise estatística e demonstra caminhos positivos para o uso de resíduos de grama no âmbito de um processo mais sustentável de gerenciamento dos resíduos sólidos orgânicos.

Apesar dos resultados obtidos sejam tão promissores, o potencial de produção de biogás pela codigestão dos resíduos é ainda muito maior, considerando-se que ele pode chegar a 500-600 Nml, como verificado na literatura. Além disso, considerando a produção de biogás por grama de sólido volátil total, tem-se que o primeiro e o segundo tratamento, em média, tiveram o mesmo desempenho ( $25 \mathrm{Nml} / \mathrm{g} . \mathrm{SV}$ ).

A baixa eficiência da produção de biogás observada no experimento realizado, pode estar relacionada à produção de ácidos graxos no processo, devido ao uso da grama e pode ser melhorado em estudos futuros. Este fato foi verificado pela baixa remoção orgânica e demonstrado pelos sólidos voláteis com remoção de aproximadamente 50\%. A digestão incompleta pode ter sido interrompida por um acúmulo de ácidos orgânicos voláteis, como mencionado, provenientes de uma maior digestibilidade dos resíduos alimentares frente aos verdes e/ou de um alto teor de carga orgânica, visualizado no pH ácido dos digeridos.

Comparada com a potencialidade de produção que se pode alcançar, tem-se que a associação dos resíduos alimentares com os verdes apresenta um favorável viés de ampla utilização, tendo em vista um contexto no qual se tem vasta oferta destes resíduos (provenientes da poda, domicílios, feiras, mercados, dentre outros) e a necessidade, sob o prisma da sustentabilidade, de redução expressiva na quantidade de 
resíduos, principalmente orgânicos que são destinados aos métodos de disposição no solo - aterros sanitários, aterros controlados e lixões (concedendo, portanto, uma destinação mais apropriada a estes resíduos.

Destarte, a digestão anaeróbia não só contribui para com a ordem de prioridade exigida pela Política Nacional de Resíduos Sólidos, como representa uma fonte de energia renovável, que se enquadra em um panorama que solicita mudanças em prol de maior segurança energética e de uma transição para uma matriz energética mais sustentável, caracteristicamente com menos emissão de carbono. Por fim, frisa-se que as potencialidades de uso da digestão anaeróbia estão presentes principalmente no fechamento de um ciclo, no qual os resíduos, que anteriormente seriam descartados sem nenhuma valorização correlata, se tornam matérias-primas para geração de energia e massa digerida com características fertilizantes.

\section{REFERÊNCIAS}

ABRELPE. Associação Brasileira de Empresas de Limpeza Pública e Resíduos Especiais. Panorama dos resíduos sólidos no Brasil: 2018/2019. São Paulo: ABRELPE, 2019.

ALVES, I. R. F. S.. Avaliação da co-digestão na produção de Biogás. Tese (Doutorado em Engenharia) - Universidade Federal do Rio de Janeiro, Rio de Janeiro, 2016.

ANDRIAMANOHIARISOAMANANA, F. J.; MATSUNANI, N.; YAMASHIRO, T.; IWASAKI, M.. High-solids anaerobic monodigestion of riverbank grass under thermophilic conditions. Journal of Environmental Sciences, v.52, p.29-38, 2017. DOI: http://doi.org/10.1016/i.jes.2016.05.005

APHA. American Public Health Association. Standard methods for the examination of water and wastewater. Washington: APHA, 2005.

ARIUNBAATAR, J.; PANICO, A.; ESPOSITO, G.; PIROZZI, F.; PIET, N. L. L.. Pretreatment methods to enhance anaerobic digestion of organic solid waste. Applied Energy, v.123, p.143-156, 2014. DOI:

http://doi.org/10.1016/j.apenergy.2014.02.035

BAIRD, C.; CANN, M.. Química ambiental. Porto Alegre: Bookman, 2001.

BEDOIĆ, R.. Green biomass to biogas: a study on anaerobic digestion of residue grass. Journal of Cleaner Production, v.213, p.700-709, 2019. DOI:

http://doi.org/10.1016/j.jclepro.2018.12.224

BEGGIO, G.; SCHIEVANO, A.; BONATO, T.; HENNEBERT, P.. Statistical analysis for the quality assessment of digestates from separately collected organic fraction of municipal solid waste (OFMSW) and agro-industrial feedstock. Should input feedstock to anaerobic digestion determine the legal status of digestate?. Waste Management, v.87, p.546-558, 2019. DOI: http://doi.org/10.1016/j.wasman.2019.02.040

BRASIL. Lei n.12.305, de 2 de agosto de 2010. Institui a Política Nacional de Resíduos Sólidos. Brasília: DOU, 2010.

BROWN, D.; JIAN SHI, Y. L.. Comparison of solid-state to liquid anaerobic digestion of lignocellulosic feedstocks for biogas production. Bioresource Technology, v.124, p.379-
386, 2012. DOI:

http://doi.org/10.1016/j.biortech.2012.08.051

BROWN, D.; LI, Y.. Solid state anaerobic co-digestion of yard waste and food waste for biogas production. Bioresource Technology, v.127, p.275-280, 2013. DOI: http://doi.org/10.1016/j.biortech.2012.09.081

CHARLES, W.; WALKER, L.; CORD-RUWISCH, R.. Effect of preaeration and inoculum on the start-up of batch thermophilic anaerobic digestion of municipal solid waste. Bioresource Technology, v.100, n.8, p.2329-2335, 2009. DOI: http://doi.org/10.1016/i.biortech.2008.11.051

CHEN, X.; YAN, W.; SHENG, K.; SANATI, M.. Comparison of high-solids to liquid anaerobic co-digestion of food waste and green waste. Bioresource Technology, v.154, p.215-221, 2014. DOI: http://doi.org/10.1016/j.biortech.2013.12.054

CHEN, Y.; CHENG, J. J.; CREAMER, K. S.. Inhibition of anaerobic digestion process: a review. Bioresource Technology, v.99, p.4044-4064, 2008. DOI: http://doi.org/10.1016/j.biortech.2007.01.057

COIMBRA-ARAÚJO, C. H.; MARIANI, L.; BLEY JUNIOR, C.; FRIGO, E. P.. Brazilian case study for biogas energy: Production of electric power, heat and automotive energy in condominiums of agroenergy. Renewable and Sustainable Energy Reviews, v.40, p.826-839, 2014. DOI: http://doi.org/10.1016/j.rser.2014.07.024

CURTO, D. M.; MARTíN, M.. Renewable based biogas upgrading. Journal of Cleaner Production, p.50-59, 2019. DOI: http://doi.org/10.1016/j.jclepro.2019.03.176

FAISAL, S.; SALAMA, E.; MALIK, K. LEE, S.; LI, X.. Anaerobic digestion of cabbage and cauliflower biowaste: Impact of iron oxide nanoparticles (IONPs) on biomethane and microbial communities alteration. Bioresource Technology Reports, v.12, 2020. DOI: https://doi.org/10.1016/j.biteb.2020.100567

FEROLDI, M.; CAROLINE, A.; NEVES, C. E. B.; ARANTES, M. K.; ALVES, H. J.. Storage of purified biogas (biomethane) at low pressure. Brazilian Archives of Biology and Technology, Curitiba, v.61, p.1-8, 2018. DOI: 
http://dx.doi.org/10.1590/1678-4324-smart-2018000014

HOLM-NIELSEN, J. B.; AL SEADI, T.; OLESKOWICZ-POPIEL, P.. The future of anaerobic digestion and biogas utilization. Bioresource Technology, v.100, n.22, p.5478-5484, 2009.

ICLEI. Governos Locais pela Sustentabilidade. Manual para aproveitamento do biogás: volume dois, efluentes urbanos. ICLEI: São Paulo, 2010.

KAWAI, M.; NAGAO, N.; TAJIMA, N.; NIWA, C.; MATSUYAMA, T.; TODA, T.. The effect of the labile organic fraction in food waste and the substrate/inoculum ratio on anaerobic digestion for a reliable methane yield. Bioresource Technology, v.157, n.11, p.174-180, 2014. DOI: http://doi.org/10.1016/j.biortech.2014.01.018

KETSUB, N.; LATIF, A.; KENT, G.; DOHERTY, W. O. S.; O'HARA, I. M.; ZHANG, Z.; KAPARAJU, P.. A systematic evaluation of biomethane production from sugarcane trash pretreated by different methods. Bioresource Technology, v.319, p.124137, 2021. DOI: https://doi.org/10.1016/j.biortech.2020.124137

KHALID, A.; ARSHAD, M.; ANJUM, M.; MAHMOOD, T.; DAWSON, L.. The anaerobic digestion of solid organic waste. Waste Management, v.31, p.1737-1744, 2011. DOI: http://doi.org/10.1016/j.wasman.2011.03.021

KWIETNIEWSKA, E.; TYS, J.. Process characteristics, inhibition factors and methane yields of anaerobic digestion process, with particular focus on microalgal biomass fermentation. Renewable \& Sustainable Energy Reviews, v.34, p.491-500, 2014. DOI: http://doi.org/10.1016/j.rser.2014.03.041

LIMA, H. Q.. Determinação de parâmetros cinéticos do processo de digestão anaeróbia dos resíduos orgânicos de Santo André - SP por meio de testes do potencial bioquímico de metano. Tese (Doutorado em Energia) Universidade Federal do ABC, Santo André, 2016.

LOPES, G. A.; MOZER, T. S.; CARLI, A. A.. Comparative analysis of biogas generation for the solid waste sector using unmanned aerial vehicle with the Brazilian model of greenhouse gas emissions in areas with no operational history. Boletim de Ciências Geodésicas, Curitiba, v.24, n.1, p.98-124, 2018.

LUCON, O.; GOLDEMBERG, J.. Crise financeira, energia e sustentabilidade no Brasil. Estudos Avançados, v.65, n.23, p.121-130, 2009.

MAO, C.; FENG, Y.; WANG, X.; REN, G.. Review on research achievements of biogas from anaerobic digestion. Renewable and Sustainable Energy Reviews, v.45, p.540555, 2015. DOI: http://doi.org/10.1016/j.rser.2015.02.032

MATTIOLI, A.; BOSCARO, D.; DALLA VENEZIA, F.; SANTACROCE, F. C.; PEZZUOLO, A.; SARTORI, L.; BOLZONELLA, D.. Biogas from residual grass: a territorial approach for sustainable bioenergy production. Waste and Biomass Valorization, v.8, p.2747-2756, 2017. DOI: http://doi.org/10.1007/s12649-017-0006-y

MAUGER, R.. Promoting public participation in the energy transition: the case of france's national debate. PER -

Potchefstroom, v.22, n.1, p.1-29, 2019. DOI: http://doi.org/10.17159/1727-3781/2019/v22i0a4290

MINITAB. Introdução ao Minitab Statistical Software. MINITAB, 2019.

ONU. Organização das Nações Unidas. ODS7: Objetivo de Desenvolvimento Sustentável 7. Brasília: ONU, 2015.

SCARLAT, N.; D., J.; FAHL, F.. Biogas: developments and perspectives in Europe. Renewable Energy, v.129, p.457472, 2018. DOI:

http://doi.org/10.1016/j.renene.2018.03.006

SCHIEVANO, A.; POGNANI, M.; D'IMPORZANO, G.; ADANI, F.. Predicting anaerobic biogasification potential of ingestates and digestates of a full-scale biogas plant using chemical and biological parameters. Tecnologia Bioresource, v.99, n.17, p.8112-8117, 2008. DOI:

http://doi.org/10.1016/j.biortech.2008.03.030

SHANE, A.; KAFWEMBE, Y.; KAFWEMBE, P.. The viability of biomethane as a future transport fuel for Zambian towns: A case study of Lusaka. Journal of Energy in Southern Africa, Cape Town, v.29, n.3, p.86-95, 2018. DOI: http://doi.org/10.17159/2413-3051/2018/v29i3a4893

SNIS. Secretaria Nacional de Saneamento. Sistema Nacional de Informações sobre Saneamento: Diagnóstico do Manejo de Resíduos Sólidos Urbanos - 2017. Brasília: SNS, 2019.

STRÖMBERG, S.; NISTOR, M.; LIU, J.. Towards eliminating systematic errors caused by the experimental conditions in Biochemical Methane Potential (BMP) tests. Waste Management, v.34, n.11, p.1939-1948, 2014. DOI: http://doi.org/10.1016/i.wasman.2014.07.018

WU, D.; LI, L.; ZHAO, X.; PENG, Y.; YANG, P.; PENG, X.. Anaerobic digestion: A review on process monitoring. Renewable and Sustainable Energy Reviews, v.103, p.1-12, 2019. DOI:

http://doi.org/10.1016/j.rser.2018.12.039

ZHANG, C.; SU, H.; BAEYENS, J.; TAN, T.. Reviewing the anaerobic digestion of food waste for biogas production. Renewable and Sustainable Energy Reviews, v.38, n.11, p.383-392, 2014. DOI: http://doi.org/10.1016/j.rser.2014.05.038

ZHU, J.; ZHENG, Y.; XU, F.; LI, Y.. Solid-state anaerobic codigestion of hay and soybean processing waste for biogas production. Bioresource technology, v.154, p.240-247, 2014. DOI: http://doi.org/10.1016/j.biortech.2013.12.045

A CBPC - Companhia Brasileira de Produção Científica (CNPJ: 11.221.422/0001-03) detém os direitos materiais desta publicação. Os direitos referem-se à publicação do trabalho em qualquer parte do mundo, incluindo os direitos às renovações, expansões e disseminações da contribuição, bem como outros direitos subsidiários. Todos os trabalhos publicados eletronicamente poderão posteriormente ser publicados em coletâneas impressas sob coordenação da Sustenere Publishing, da Companhia Brasileira de Produção Científica e seus parceiros autorizados. Os (as) autores (as) preservam os direitos autorais, mas não têm permissão para a publicação da contribuição em outro meio, impresso ou digital, em português ou em tradução. 